\title{
Premixed-flame oscillations in narrow channels
}

\author{
Daniel Martínez-Ruiz \\ ETSIAE, Departamento de Mecánica de Fluidos y Propulsión Aeroespacial, \\ Universidad Politécnica de Madrid, Madrid 28040, Spain \\ Fernando Veiga-López and Mario Sánchez-Sanz ${ }^{\dagger}$ \\ Departamento de Ingeniería Térmica y de Fluidos, Universidad Carlos III de Madrid, \\ Leganés 28911, Spain
}

(Received 19 January 2019; published 24 October 2019)

\begin{abstract}
This paper is associated with a video winner of a 2018 APS/DFD Milton van Dyke Award for work presented at the DFD Gallery of Fluid Motion. The original video is available online at the Gallery of Fluid Motion, https://doi.org/10.1103/APS.DFD.2018. GFM.V0018
\end{abstract}

DOI: 10.1103/PhysRevFluids.4.100503

Propagation of premixed flames in confined vessels typically undergoes oscillatory motions due to the coupling of the reaction front, the induced flow dynamics, and acoustics. The first scientific report regarding thermoacoustics [1] appeared over two centuries ago and, because of its practical importance, great effort has been done since then to understand the phenomenon [2]. However, most of the experimental research has been performed in vertical tubes [3] under strong buoyancy effects. Here, the first thermoacoustic study reported in a horizontal narrow-channel (Hele-Shaw) vessel, as described in [4], has proved instrumental to further understand the dynamics of the instability.

First, a top-view composition of a lean flame with equivalence ratio $\phi=0.8<\phi_{\text {cr }}$ and a rich one with $\phi=1.2>\phi_{\mathrm{cr}}$ is shown in Fig. 1 . The parameter $\phi_{\mathrm{cr}}$ indicates the critical equivalence ratio at which the flame transitions from the primary acoustic regime, experiencing small-amplitude oscillations and acoustic waves of the order of hundreds of Pascals, to the secondary acoustic instability, encountering large-amplitude oscillations with a characteristic finger-shaped front and pressure waves one order of magnitude higher.

In addition, a detail of the evolution from primary oscillations to the secondary regime is shown in Fig. 2, offering unprecedented lateral perspectives of the flame front during successive cycles of the aforementioned process. In column (a), shortly after ignition, the flame propagates experiencing smooth oscillations and maintaining a parabolic front, before evolving to a nearly planar flame (b). Next, the flame develops a rippled front, with a cell wavelength smaller than the separation between plates $h=10 \mathrm{~mm}$ (c). These new cells progressively wrinkle the front and the flame quickly increases its oscillation amplitude (d) that finally undergoes a violent beat (e)

\footnotetext{
*Also at Fluid Mechanics Group, Universidad Carlos III de Madrid; daniel.mruiz@upm.es

†http://www.fluidosuc3m.es

Published by the American Physical Society under the terms of the Creative Commons Attribution 4.0 International license. Further distribution of this work must maintain attribution to the author(s) and the published article's title, journal citation, and DOI.
} 


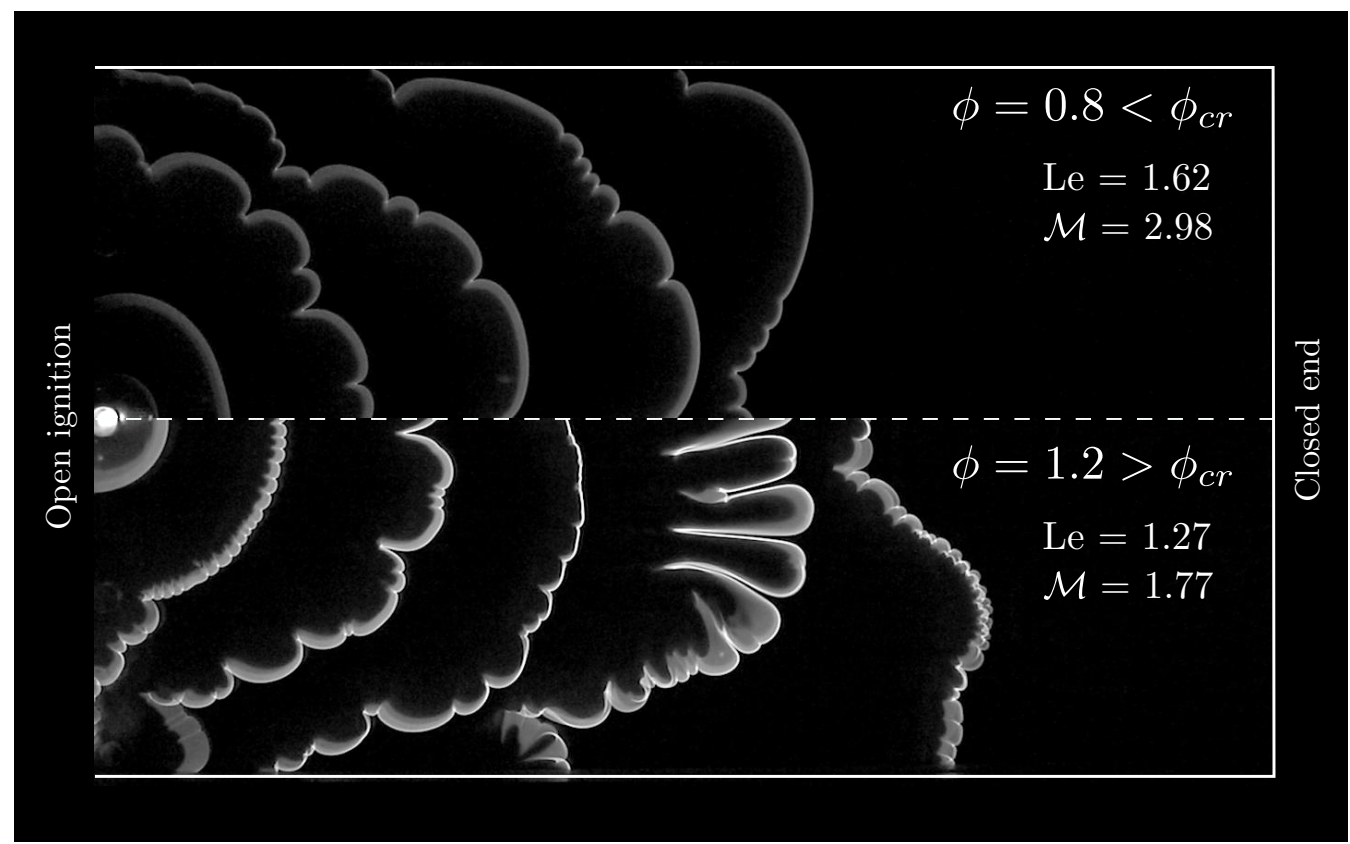

FIG. 1. Top view of premixed propane-air flames propagating in a 900-mm-long, 500-mm-wide, and 10-mm-thick vessel open at the ignition end. https://doi.org/10.1103/APS.DFD.2018.GFM.V0018

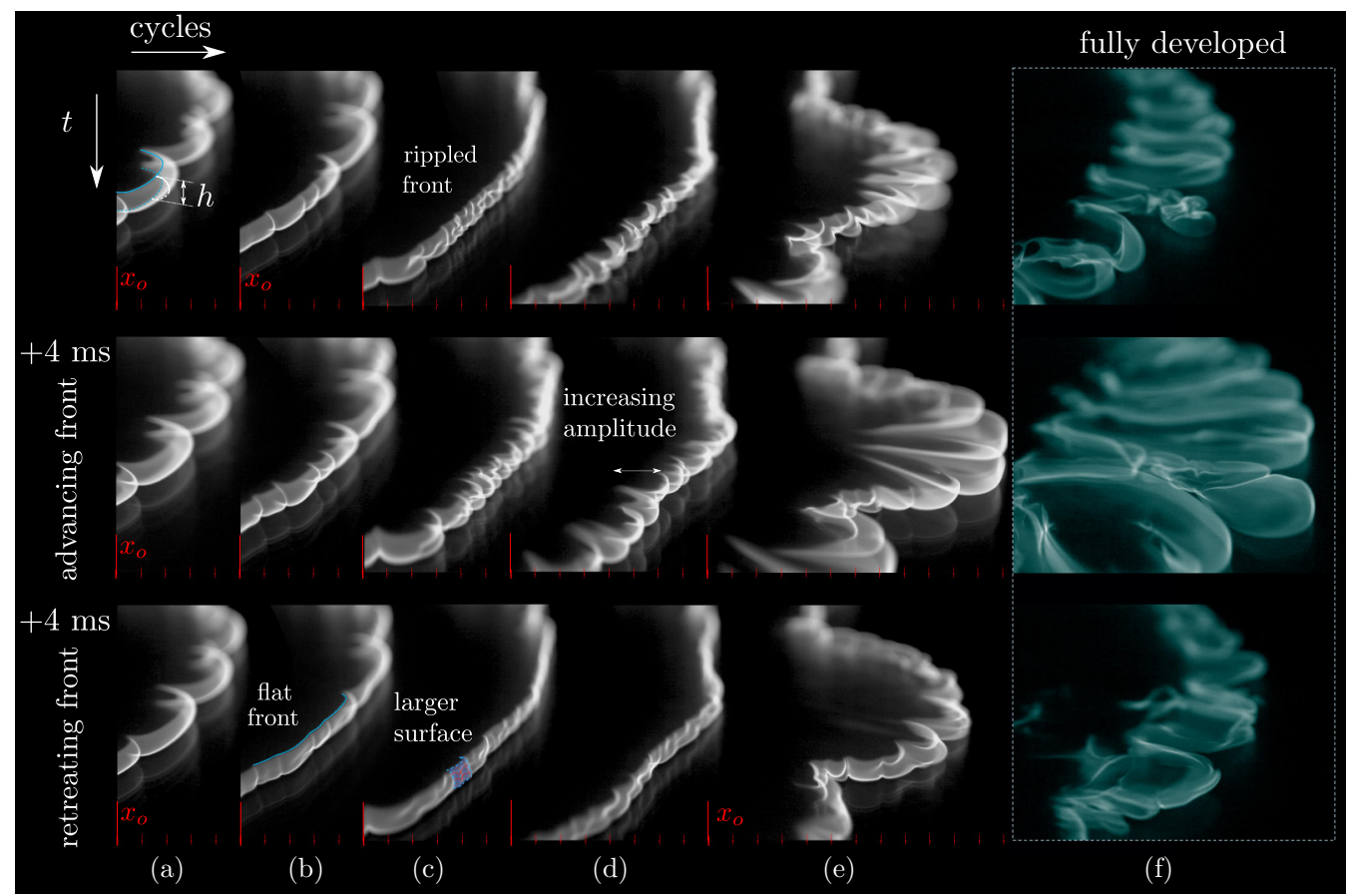

FIG. 2. Side-view detail of the instability transition. Columns (a)-(e) show the reactive front at the beginning, peak, and trough of each cycle. Red vertical lines determine the origin of the picture $x_{o}$ (long) and spatial references (short). Column (f) shows fully developed secondary oscillations at a different location. https://doi.org/10.1103/APS.DFD.2018.GFM.V0018 
at a frequency near $100 \mathrm{~Hz}$ and velocities of around $10 \mathrm{~m} / \mathrm{s}$. Fully developed instabilities display complex dynamics during the late stages of the propagation (f). Although elaborate discussions on thermodiffusive parameters (effective Lewis number $L e$ ), front curvature and strain (Markstein number $\mathcal{M}$ ), equivalence ratio $(\phi)$, and acoustic effects have been previously offered in the literature, the exact physical mechanism responsible for the transition remains unsolved.

The authors acknowledge financial support from the Spanish Ministry of Economy and Competitiveness under project ENE2015-65852-C2-1-R (MINECO/FEDER, UE). We thank M. Santos, I. Pina, and D. Díaz for their advice in the design and construction of the experimental setup.

[1] B. Higgins, On the sound produced by a current of hydrogen gas passing through a tube, J. Nat. Philos., Chem. Arts 1, 129 (1802).

[2] T. Poinsot, Prediction and control of combustion instabilities in real engines, Proc. Combust. Inst. 36, 1 (2017).

[3] G. Searby, Acoustic instability in premixed flames, Combust. Sci. Technol. 81, 221 (1992).

[4] F. Veiga-López, D. Martínez-Ruiz, E. Fernández-Tarrazo, and M. Sánchez-Sanz, Experimental analysis of oscillatory premixed flames in a Hele-Shaw cell propagating towards a closed end, Combust. Flame 201, 1 (2019). 